\title{
Research of Particles Distribution Function in Horizontal Liquid-Solid Circulating Fluidized Bed
}

\author{
WU Jing ${ }^{1}$, WANG Jiang-tao ${ }^{2}$, LIU Yan ${ }^{2}$ \\ ${ }^{1}$ School of Biological and Environmental Engineering, Tianjin Vocational Institute, Tianjin 300410, \\ China \\ ${ }^{2}$ College of Chemical Engineering, Hebei University of Technology, Tianjin 300130, China
}

Keywords: liquid-solid circulating fluidized bed; Kenics; particle distributor

Abstract: A horizontal liquid-solid multi-tube circulating fluidized bed was designed to study the influence of front header distributor, liquid velocity,Kenics static mixer to various particle's distribution and discuss the pressure of front header to back header. The CCD image measurement and data processing system was used to study the particle distribution and the U-tube differential was used to measure the pressure of front header to back header. Experimental results show that the front header distributor can improve the particle distribution in the tube bundle of the fluidized bed;the particle distribution will be best in the tube when the liquid velocity is $0.6 \mathrm{~m} / \mathrm{s}$ and Kenics static mixer is in the tube; in the same condition,plastic particles distribution is better than corundum ball particles distribution.

\section{Introduction}

Heat-exchanger equipment is an important operation unit widely used in Petroleum, Chemicals, Bio-pharmaceutical, Sewage treatment and other sectors. However, different levels of fouling were happened frequently on the heat-transfer surface of heat-exchanger equipment. Especially involving heating process of evaporation, fouling status is more serious and obstructs to heat transfer and mass transfer. Therefore, many scholars ${ }^{[1 \sim 5]}$ put inert gas particles into heat-exchanger equipment to prevent and remove fouls and enhance heat transfer factor. For horizontal liquid-solid multi-tube circulating fluidized bed, the distribution of particle in pipe bundle is very non-homogeneous. When the density of particle is greater than the density of liquid, particle must be precipitated after flowing a section of distance in pipe. It will lead pipe blocked and even the entire device blocked in the end. In this paper, the front header of horizontal liquid-solid circulating fluidized bed has been reformed and do research of particle distribution status in pipe bundle.

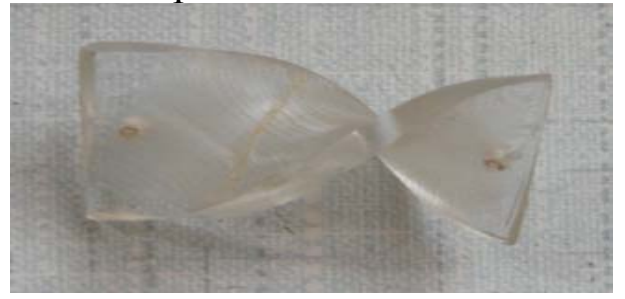

Fig.1 Kenics static mixer

Static mixer is a kind of energy-saving equipment which structure is very simple and compact. It is very easy to produce and install, and the pressure drop is also very low. This kind of equipment can realize cutting, mixing of fluid repeatedly. It can also be chemical process and intensification. It even has the trend to replace the agitation reactor on many occasions. Therefore, this article will would introduce the Kenics static mixer(Fig.1) which widely used and made at the earlier time to Horizontal tube liquid-solid circulating fluidized bed to improve the particle distribution inside the pipe ${ }^{[6 \sim 8]}$. 


\section{Experiment}

\subsection{Experimental Procedures}

Experimental flow is shown in Figure 2. The whole experiment device consist of water tank, centrifugal pump, electromagnetic flowmeter, liquid-solid separator, horizontal multi-tube heat exchanger, CCD image measurement and data processing system and circulation pipes, etc. Water from water tank is pumped into circulation system by centrifugal pump. Use valves to control flow rate. Solid particle is added into the system through the liquid-solid separator. Particle and water was mixed and then entry front header. Particle and water will entry horizontal pipe bundle evenly with the action of front header distributor. Then liquid-solid two-phase flow through the circulation pipes into liquid-solid separator to do Solid-Liquid Separation. Particle entries the circulation system again and water returns to water tank.

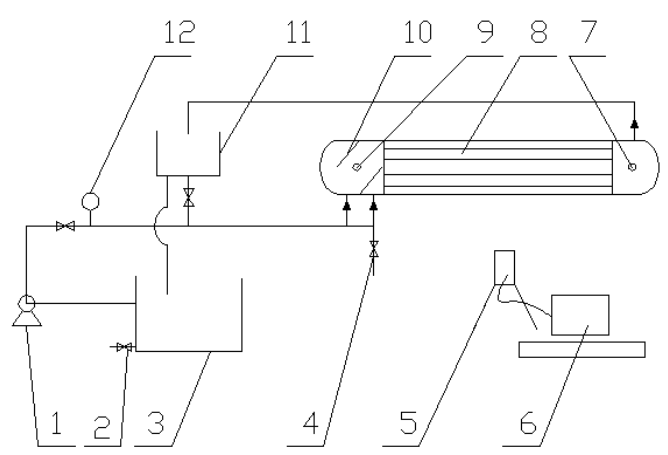

Fig.2 Schematic diagram of experimental flow 1.Centrifugal pump 2.Drain outlet 3.Water tank 4.Particle outfall 5.CCD camera 6. Computer 7,9. Pressure-measuring opening 6.8. Horizontal pipe bundle 10. Front header distributor 7.11. Liquid-solid separator 12. Electromagnetic flowmeter

\subsection{Experimental Equipment}

In this experiment, $\varphi 25 \times 3-\mathrm{L}=1000 \mathrm{~mm}$ lucite tube is used as heat-exchange tube of heat-exchanger, and inner diameter of the header is $\varphi 280 \mathrm{~mm}$. The distribution of pipe bundle is shown in Figure 3. The tubes are arranged on triangular pitch. In this experiment, in order to measure the distribution of particle in different vertical heights in tube, select D1, D2, D3 and D4 as test tubes and do image measurement and data processing at the bottom of heat-exchange pipe bundle. Refer to previous test results of single- tube, this experiment uses the Kenics static mixer which Torsion (components of the ratio of length to diameter) $Y=3.0$ and made of lucite. Installation location of Kenics static mixer is at the entrance of heat-exchange pipe bundle. Pressure measurement points are respectively at the middle of front header distributor and back header distributor to measure the pressure drop of the whole heat exchanger. Pressure Measuring Unit is U-tube manometer. In this experiment, liquid medium is tap water at room temperature. The density of water is $1000 \mathrm{~kg} / \mathrm{m}^{3}$ and viscosity is 1.0 $\mathrm{mPa} \cdot \mathrm{s}$. The properties of particles are shown in Table 1 . The volume fraction of solid particles is $3 \%$. The flow meter is electromagnetic flowmeter and the max measuring range is $20 \mathrm{~m}^{3} / \mathrm{h}$. In this experiment, the flow of liquid volume is respectively $5 \mathrm{~m}^{3} / \mathrm{h}, 7 \mathrm{~m}^{3} / \mathrm{h}, 9 \mathrm{~m}^{3} / \mathrm{h}$ and $11 \mathrm{~m}^{3} / \mathrm{h}$.

\subsection{The reforming of front header}

In the horizontal liquid-solid multi-tube circulating fluidized bed, the distribution of particle in front header is inhomogeneous due to gravity. This will lead to particles that can not entry horizontal heat-exchange pipe bundle evenly. In this paper, the front header of heat exchanger has been reformed to improve this situation and the reformed front header is shown in Figure 4. The entrances of liquid are respectively entrance 1 and entrance 2 . This can effectively avoid accumulation of solid particles in the bottom of front header. In order to let solid particle entry horizontal heat-exchange pipe bundle evenly, this experiment adds two baffles in front header. The experimental results 
demonstrate a better distribution of particle has been obtained when the angle parameters of the baffle with horizontal is $60^{\circ}$. As the result, the inclination angle of baffle $\alpha$ in this experiment is $60^{\circ}$.

Table.1 The properties of particle

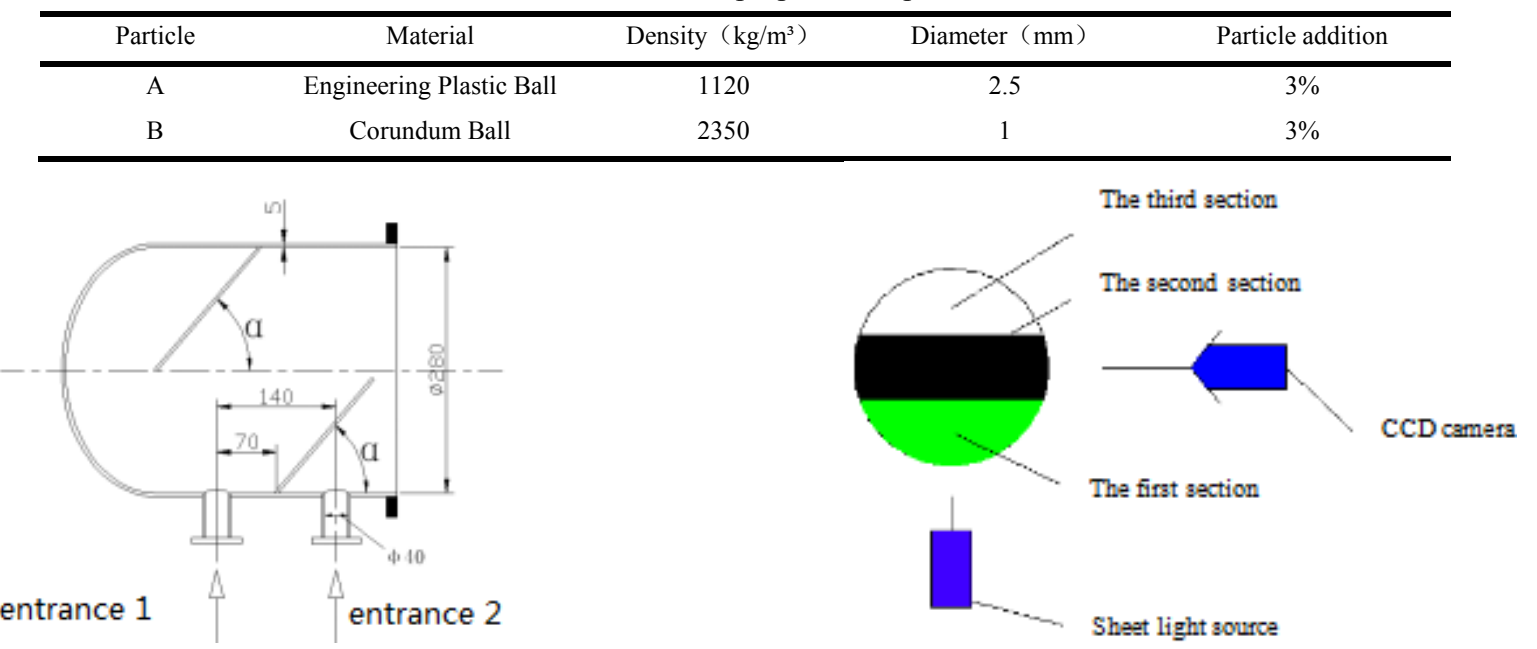

Fig.4 The reformed front header

Fig.5 Sketch of subarea for the test pipe

\section{Experiment data processing}

The distribution of particles in heat-exchange tube is inhomogeneous due to gravity. In this experiment, the inside diameter of heat-exchange tube is small but the diameter of particle is big. In this situation, this experiment divides the sheet light source direction of test pipe into three equal zones which is shown in Figure 5. The sheet light source aligns the central of subarea. Use CCD camera to read the number of particles in each subarea. Repeat several times, and then calculate the average particle number of this area. Use Formulas (1) to calculate the solid content of particles for each area. Use Formulas (2) to calculate the solid content of particles for different test pipes.

$$
\begin{gathered}
\varepsilon_{\mathrm{si}}=\overline{\mathrm{n}} * \mathrm{~S}_{\mathrm{k}} / \mathrm{S}_{\mathrm{q}} \\
\mathcal{E}_{\mathrm{i}}=\varepsilon_{\mathrm{s} 1+}+\mathcal{E}_{\mathrm{s} 2}+\mathcal{E}_{\mathrm{s} 3}
\end{gathered}
$$

$\varepsilon_{\mathrm{si}}$ - Solid content of particles for different areas

$\overline{\mathrm{n}}$ - Average particles number of this area

$\mathrm{S}_{\mathrm{k}}$ - Projected area of particles

$\mathrm{S}_{\mathrm{q}}-$ Cross-sectional area of this area

$\varepsilon_{\mathrm{i}}$ - Solid content of particles for different test pipes.

\section{Discussion on experimental result}

\subsection{Pressure drop}

In liquid-solid circulating fluidized bed, pressure drop is an expression o energy loss,which is incurred by liquid-solid two-phase flow overcoming internal friction, turbulence and mutual collision. And the energy loss inevitably accompanies with economic losses, thus pressure drop of fluidized bed is a key point in our experimental study. 


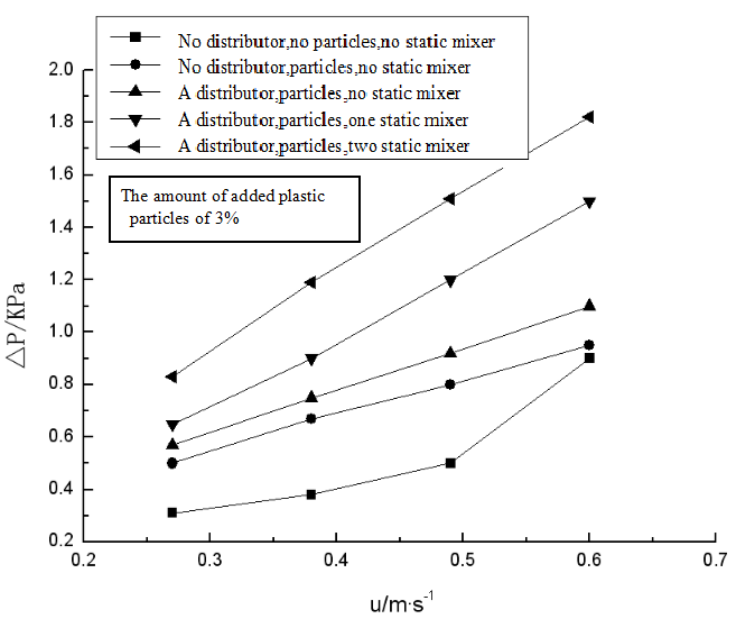

Fig.6 Adding plastic particles, pressure drop of heat exchanger under various conditions

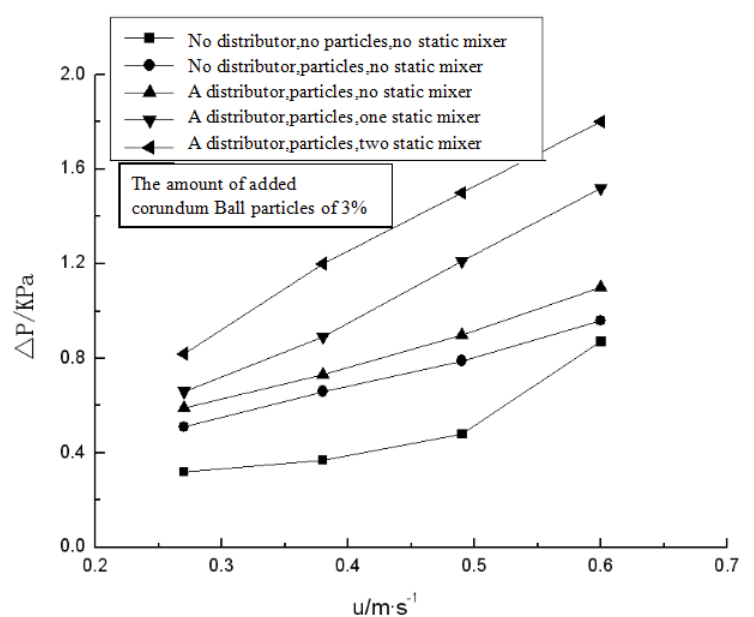

Fig.7 Adding corundum ball particles, pressure drop of heat exchanger under various conditions

Figure 6 and Figure 7 are the changing of heat exchanger pressure drop with the flow rate of the liquid in the tube under different conditions. From Figure 6 and Figure 7, we can see the factors which affect pressure drop are distributor, solid particles, static mixer, the amount of added solid particles and flow rate etc. The pressure drop achieves maximum with a distributor, particles, static mixers and greater flow rate when the same amount of particles is added. However, the type of solid particle change has a little influence to the pressure drop. This is because the mutual collision among particles is intense and loss energy when fluid flows through particle with a distributor,particles and static mixers. As the result, the pressure drop increase. The greater flow rate is, the more serious the fluid turbulence is, and so the greater the pressure drop is. The maximum pressure drop value is $2.07-3.24$ times of minimum pressure drop value with certain flow rate under different conditions.

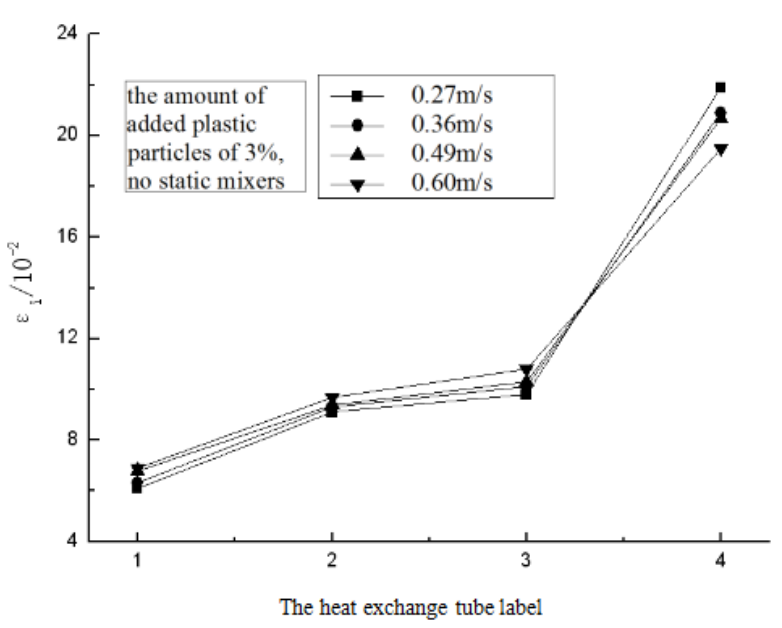

Fig.8 No distributor, plastic particles distribution at different flow rates in the pipe bundle

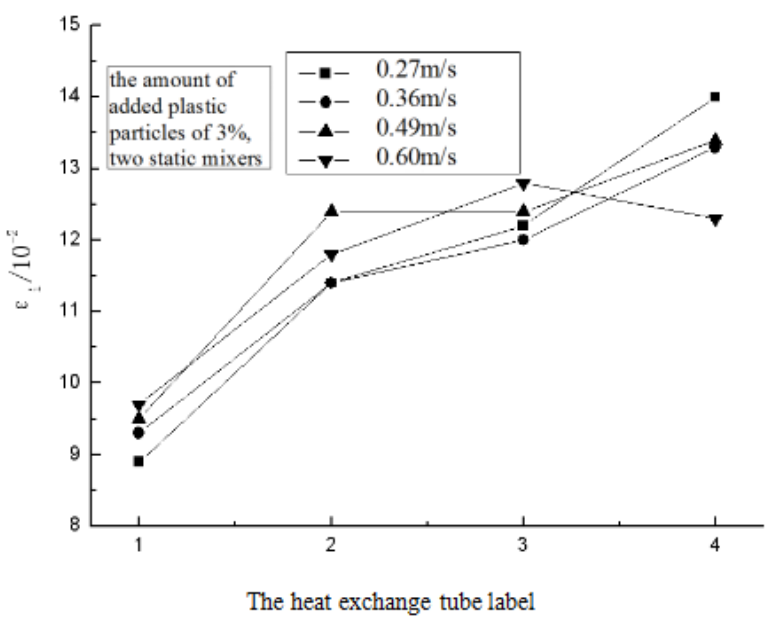

Fig.9 Two distributors, plastic particles distribution at different flow rates in the pipe bundle

\subsection{Influence of front header distributor on particles distribution in pipe bundle}

Due to the gravity action, particles distribution is uneven when entering pipe bundle from front header. Quantity of particles in the upper pipe is less than those in the lower pipe. So in this article, front header of heat exchanger is transformed. Figure 8 and Figure 9 show the distribution of particles in horizontal pipe bundle before and after the front header distributor is transformed.

It can be seen from Figure 8 and Figure 9 that the greater the flow rate is, the little the particles distribution unevenness is under certain conditions. The particles distribution unevenness has improvement in certain extent after the front header distributor is added. Particles distribution with two distributors is more even than that with one distributor, which is due to the following reason: the flow pass of liquid is changed after the distributor is added, resulting in particles being stirred by the 
fluid in front header to enter the horizontal pipe bundle more evenly. The greater the flow rate is, the more serious the writhe is, so the more even the particle distribution is.

\subsection{Influence of Kenics static mixer on in-pipe particles distribution}

Table 2 and Table 3 show the change of distribution unevenness between plastic particles and corundum ball particles before and after the Kenics static mixer is installed with the flow rate of $0.6 \mathrm{~m} / \mathrm{s}$ and the amount of added solid particles of $3 \%$.

\begin{tabular}{|c|c|c|c|c|c|c|c|c|c|c|c|c|}
\hline \multirow{2}{*}{ the solid holduper } & \multicolumn{3}{|c|}{ No 1 tube, $10^{-2}$} & \multicolumn{3}{|c|}{ No 2 tube, $10^{-2}$} & \multicolumn{3}{|c|}{ No 3 tube, $10^{-2}$} & \multicolumn{3}{|c|}{ No 4 tube, $10^{-2}$} \\
\hline & One & two & three & one & two & three & one & two & three & one & two & three \\
\hline No static mix & 5.3 & 4.3 & 2.7 & 5.8 & 3.6 & 2.5 & 6.1 & 4.2 & 2.5 & 6.4 & 3.5 & 2.4 \\
\hline One static mix & 3.5 & 3.4 & 3.4 & 4.1 & 4.0 & 3.9 & 4.2 & 4.2 & 4.1 & 4.0 & 3.9 & 3.8 \\
\hline Two static mixs & 3.0 & 2.8 & 2.7 & 4.0 & 4.0 & 3.9 & 3.9 & 3.8 & 3.9 & 4.2 & 4.1 & 4.1 \\
\hline
\end{tabular}

\begin{tabular}{c|cccccccccccccc}
\multicolumn{1}{c}{ Table.3 The impact of Kenics static mixer on corundum ball particles distributionwhen the liquid velocity is $0.6 \mathrm{~m} / \mathrm{s}$} \\
\hline the solid
\end{tabular}

It can be concluded from Table 2 and Table 3 that the distribution of in-pipe particles has great improvement after the Kenics static mixer is installed, and the distribution of plastic particles is better than corundum ball particles under the same conditions, which is due to the following reasons: when fluid flows pass through Kenics static mixer, the rotation turbulence incurred by fluid is strengthened, in this way, particles affected by "rotary force" of fluid within rotation area are redistributed. The density of corundum ball particles being far larger than that of plastic particle,so the distribution of plastic particles is better than corundum ball particles under the same conditions.

\section{Conclusion}

1. The distribution of particles in heat exchange pipe bundle has been obviously improved after the particle distributor is installed in front header.

2. Distribution unevenness of particles in heat exchange pipe is obviously decreased after Kenics static mixer is installed. With liquid flow rate becoming greater, the particle distribution unevenness decreases.

3. The distribution of plastic particles is better than corundum ball particles under the same operation condition.

\section{Reference}

[1] C. Xu, M. Han, S. Chen, J. Wang, Y. Jin, Reactor modeling of innovative

liquid-solid circulating moving bed for the synthesis of linear alkyl benzenes, Chem. Eng. Commun. 191 (2004) 796-812.

[2] S. Roy, A. Kemoun, M.H. Al-Dahhan, M.P. Dudukovic, Experimental investigation of hydrodynamics in a liquid-solid riser, AIChEJ 51 (2005) 802-835.

[3] Y. Zheng, Radial particle profiles in a liquid-solid CFB with varying viscosity , Chem. Eng. Technol . 27 (2004) 769-776.

[4] Liu, Y., Wang, Q., Zhao, B., Zhang, S. F., The Performance Research of Distributor in Liquid-solid Circulating Fluidized Bed Heat Exchanger[J]. JOURNAL OF HEBEI UNIVERSITY OF TECHNOLOGY. 2006, 35(6):18-24. 
[5] Zhang, S. F., Zhang, W., Liu, Y., Effects of the Spiral Flow Generator on Particles Distribution in Liquid-solid Horizontal Circulating Fluidized Bed[J], JOURNAL OF HEBEI UNIVERSITY OF TECHNOLOGY. 2006，2009，38(2):69-73

[6] Chen, L. B., Nie, X., and Pan, H. C., Pressure drop researches for Kenics static mixer at high Reynolds number[J], MECHANICAL \& ELECTRICAL ENGINEERING MAGAZINE, 2009, 26(12):108-111.

[7] Tu, S., D., Wang, Z., D., Gu, B., L., Development of Progress Equipment Technology in 21st Century, CHEMICAL INDUSTRY AND ENGINEERING PROGRESS, 2003,23(3):258 266

[8] Zhu, S. L., and Sun, J. H., Research of Kenics static mixer used in enhancing heat transfer of pipe [J], Chemical Engineering, 1987(3): 15-22. 